\title{
Study on Electromagnetic Shielding of Infrared /Visible Optical Window
}

\author{
Sun Yan-Jun ${ }^{1}$, Chang Hao ${ }^{1}$, Wu Song-hang ${ }^{1}$, Leng Yan-Bing ${ }^{1} \&$ Wang Li $^{1}$ \\ ${ }^{1}$ Changchun University of Science and Technology, Changchun, China \\ Correspondence: Sun Yan-Jun, Changchun University of Science and Technology, 7089 Wei-xing Road, \\ Changchun, Jilin, China. Tel: 86-431-8558-3294. E-mail: custsun@126.com
}

Received: July 23, 2015

Accepted: September 25, 2015

Online Published: November 30, 2015

doi:10.5539/mas.v9n13p231

URL: http://dx.doi.org/10.5539/mas.v9n13p231

The research is financed by (natural science foundation of Jilin Province).

\begin{abstract}
In allusion to electromagnetic radiation damage that existed in daily life, social safety and military field, electromagnetic shielding technology of infrared and infrared optical window was studied. Transparent conductive film mesh was proposed in optical window, the mesh is composed of oxide films including ITO, $\mathrm{SnO}_{2}$ etc, for decreasing infrared and visible low transmission problem of traditional metal mesh. The effects of photoelectric properties comes from mesh line width and cycle was analysised in this paper, different line width and cycle ITO film mesh was manufactured, by the way of coating、 lithography and chemical erosion method. The Comparison Study of ITO film mesh and metal mesh, we concluded that infrared transmission of ITO mesh is higher than metal mesh, and even more than $10 \%$; that shielding efficiency of ITO mesh is lower than metal mesh, but the gap is less, only $2 \sim 3 \mathrm{~dB}$. So it has practical value by ITO mesh instead of metal mesh.
\end{abstract}

Keywords: shielding efficiency, infrared transmission, mesh, ITO

\section{Introduction}

Modern electronic equipment to social life bring convenience, comfort, while doubling its power equipment, a substantial increase in electromagnetic radiation on the ground, it has reached the degree of impact on human health. Criminals using electromagnetic technology for monitoring, surveillance, spying, so privacy and state secrets have been threatened. In addition, the electromagnetic radiation also cause a great harm in the military field, in order to achieve military precision strikes guided missiles and other weapons, often using infrared, visible, TV and other controlled guidance system, and interfere with electromagnetic radiation often make weapons guidance system failure, while taking advantage of this phenomenon, the opposing sides will use electromagnetic interference electronic warfare, causing the enemy communication systems paralyzed. Therefore, the study of a shorter wavelength of visible light, infrared light transmission efficiency, and longer wavelength electromagnetic wave shielding technology to eliminate or reduce the hazards of electromagnetic radiation caused significant meaning.

Military currently uses metal mesh technology shielding visible, unwanted electromagnetic wave of infrared window, but the biggest shortage of a metal mesh is in the long-wave electromagnetic radiation shield, while the visible and infrared also a large block. Practice has proved that some of the metal mesh is better shielding, visible and infrared transmission maximum loss of $30 \%$.

This article proposes to solve this problem using ITO transparent film mesh instead of metal mesh shield method, ITO transparent film mesh is based on ITO transparent film, made into a mesh structure by the way of lithography and chemical erosion method. ITO film is an oxide film, an ITO film is preferably plated sheet resistance which up to $5 \Omega / \square$ or less, at or near the level of the metal thin film, without regard to the substrate, its visible light transmission rate of more than 98 percent, infrared transmission up to $60 \%$, while the metal with the same surface resistance of the film its visible and infrared transmission is almost zero. Therefore ITO transparent film mesh same structure its visible and infrared transmission will far exceed the metal mesh. 


\section{The Electromagnetic Shielding Efficiency of Mesh}

Conductive mesh structure shown in Figure 1. It is a mesh structure bya plurality of lines formed perpendicular to each other For ease of calculation, the mesh line width set for the $2 \mathrm{a}, \mathrm{g}$ is a mesh line cycle.
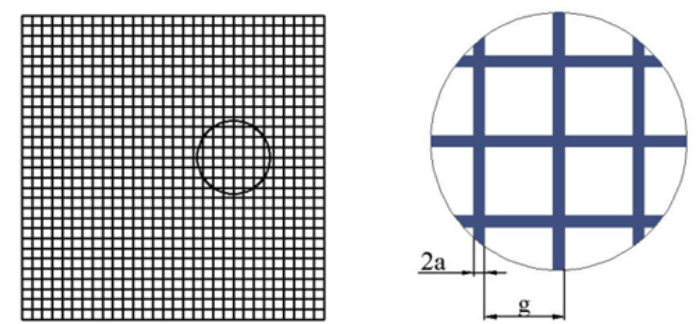

Figure 1. Structure schematic diagram of mesh

The actual mesh for shielding, thickness $t$ is much smaller than the width $2 a$, width $2 \mathrm{a}$ far less than the width of the line cycle g, for vertically incident electromagnetic wave shielding effect has normalized admittance, its value is ${ }^{[3]}$ :

$$
y=f_{0}\left[\ln \left(\sin \frac{\pi a}{g}\right)\right]\left(\frac{f}{f_{0}}-\frac{f_{0}}{f}\right)^{-1}
$$

Where: $\mathrm{f} 0$ is the resonance frequency. To achieve long-wave electromagnetic shielding, requiring the wave length $\lambda$ much larger than g, normalized admittance is approximately equal to:

$$
y=-g / \lambda \ln \left(\sin \frac{\pi a}{g}\right)
$$

The transmission of mesh is directly related to admittance, the transmission of electromagnetic waves at normal incidence when separate conductive mesh is:

$$
T_{\text {电 }}=4 y^{2} /\left(1+4 y^{2}\right)
$$

According to the equivalent film theory, in the electromagnetic wavelength $\lambda$ greater than the mesh period $g$, an independent guide mesh transmission mesh is equivalent to a transmission same refractive index ne and the same thickness $d$ of the film has no absorption. Conductive mesh transmission can also be equal to the following formula:

$$
T_{\square}=\frac{4}{2+2 \cos ^{2} \beta_{\mathrm{e}}+\left[\left(n_{\mathrm{e}} / n_{\mathrm{o}}\right)^{-2}+\left(n_{\mathrm{e}} / n_{\mathrm{o}}\right)^{2}+2\right] \sin ^{2} \beta_{\mathrm{e}}}
$$

Where: ne is the refractive index equivalent film; no refractive index of the incident medium; $\beta$ e is equivalent phase thickness of the film. For the conductive mesh is, $\cos \theta \approx 1$ and ne $=1, n p=n s=n e$, at any angle and therefore the electromagnetic wave incident on the shielding effectiveness of the impact phase conductive mesh it is very small. Electromagnetic shielding effectiveness is expressed in decibels, the $\mathrm{T}$ logarithmic values of the shielding efficiency:

\section{Infrared Transmission of Mesh}

$$
S=-10 \lg T_{\text {electric }}
$$

Traditional metal mesh screen visible and infrared light transmission of great influence, in the form of energy can calculate the transmission ratio is approximately equal obscuration ratio $^{[4]:}$

$$
T_{\text {metal }}=\frac{(g-2 a)^{2}}{g^{2}}
$$

The mesh formed by the transparent conductive film, with high visible light transmission, visible light transmission the requirements of the window without regard. Although infrared light absorption is large, but in itself it is higher than the transmission of the metal, its infrared transmission:

$$
T=\text { T metal }+(1-\text { T metal }) \text { T transmission }
$$

Where: $\mathrm{T}$ transmission to infrared waves transmission. If mesh for an imaging system, the infrared imaging 
should be based on diffraction theory calculations to take advantage of scalar diffraction theory of optical transmission attenuation system analysis, the transmission of expression is ${ }^{[5]}$ :

$$
t(x, y)=\left[\operatorname{rect}\left(\frac{x}{g-2 a}, \frac{y}{g-2 a}\right) * * \sum \sum \delta(x-n g, y-m g)\right] \times \operatorname{rect}\left(\frac{x}{N g}, \frac{y}{N g}\right)
$$

Where: $\mathrm{N}$ is the number of incident light grid; $\mathrm{n}, \mathrm{m}$ is an integer sequence; rect $(\mathrm{x}, \mathrm{y})$ is a two-dimensional rectangular transmission function. Peak value is equal to the incident light reflected by a metal mesh generated complex diffraction sequence, with and without mesh optical system point spread function, then the sequence is a sequence of any diffraction percentage point spread function of the ratio of energy:

$$
\frac{E(n, m)}{E}=k\left(\frac{g-2 a}{g}\right)^{4} \sin c^{2}\left[\frac{n(g-2 a)}{g}\right] \cdot \sin c^{2}\left[\frac{m(g-2 a)}{g}\right]
$$

Where: $\mathrm{k}(1 \sim 1.5)$ is coefficients, and transmission of the transparent conductive film red concerned. Center zero diffraction order sequence is effective, while the other level is small compared with the central zero-order effect on the image, substantially negligible, then the center of the zero order light transmission is:

$$
T(0,0)=k \frac{E(0,0)}{E}=k\left(\frac{g-2 a}{g}\right)^{4}
$$

\section{Experiment}

To verify the transparent conductive film and the infrared transmission mesh shielding effect, it produced a number of pieces of different width and cycle of the transparent conductive film mesh and metallic mesh comparative tests, the transparent conductive film ITO film using more common. ITO film mesh production take coating, lithography and chemical erosion method, the specific process shown in Figure 2.

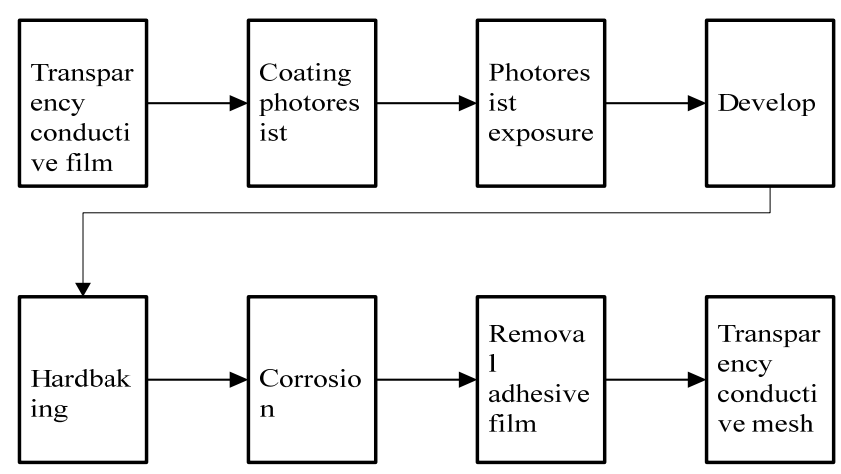

Figure 2. Manufacturing process of transparent conductive mesh

In the experiment, ITO transparent conductive film by DC magnetron sputtering system technology that uses plasma in $\mathrm{Ar}-\mathrm{O}_{2}$ mixed atmosphere at alternating electric field and magnetic field effect, so that the acceleration of energetic particle bombardment In-Sn alloy target, target surface atoms from the lattice $\phi 100 \mathrm{~mm}$ and transferred to the surface of a quartz substrate forming the film; Using centrifugal spin coating ITO film surface after the coating thickness $1 \mu \mathrm{m}$ negative photoresist, coating requires baking the plastic surface drying; Lithographic exposure process using BOL500 laser direct writing system, adjust the focal lithography objective spot reach grid line width; The use of negative resists dedicated developer make unexposed photoresist dissolved, to give a mesh structure formed by the photoresist; The substrate was immersed in aqua regia consisting of sulfuric acid and nitric acid etching, the resist is removed without protection ITO film, in addition to the remaining photoresist after etching solution to obtain ITO transparent conductive film is formed by the gate. In order to analyze mesh and infrared transparent electromagnetic shielding effect, the experiment produced a large sample of different structural parameters. The transparent conductive film is different from the metal mesh grid, due to the high visible light transmission and can not be directly observed with a microscope to test mesh shape structure, optical and electrical properties when testing is complete to give a layer of nickel plating technology using film on the surface of the system, obtained micrograph shown in Figure 3. 


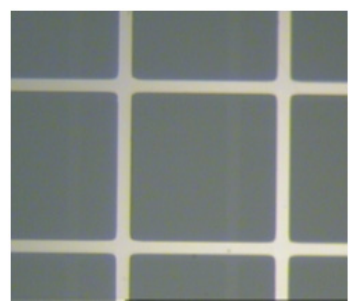

Figure 3. Microscopy photo of ITO mesh nickel plating

\section{Result Analysis}

\subsection{Transparent Conductive Mesh Width Effect the Photoelectric Property}

Radar frequency range between $2 \sim 18 \mathrm{GHz}$, infrared imaging band of $3 \sim 14 \mu \mathrm{m}$. Experiment selected $18 \mathrm{GHz}$ radar waves and infrared waves $4.6 \mu \mathrm{m}$ electromagnetic shielding and infrared transmission capability studies to verify the ITO transparent conductive mesh shielding and through, but will make the same width and cycle compared metal mesh the study. Experimental production mesh period $\mathrm{g}=300 \mu \mathrm{m}$, width respectively $1 \sim 12 \mu \mathrm{m}$ two mesh test. A quartz substrate infrared transmission of $95 \%$. The test results are shown in Table 1. Figure 4 and Figure 5 is a visual graph data table for the description.

Table 1. Different ling width shielding efficiency and infrared transmission

\begin{tabular}{lcccc}
\hline $\begin{array}{c}\text { Line width/ } \\
\mu \mathrm{m}\end{array}$ & $\begin{array}{c}\text { ITO mesh Infrared } \\
\text { transmission } /(\%)\end{array}$ & $\begin{array}{c}\text { ITO mesh shielding } \\
\text { efficiency/dB }\end{array}$ & $\begin{array}{c}\text { Metal mesh Infrared } \\
\text { transmission } /(\%)\end{array}$ & $\begin{array}{c}\text { Metal mesh shielding } \\
\text { efficiency/dB }\end{array}$ \\
\hline 1 & 94.4 & 14.3 & 94.1 & 14.5 \\
2 & 92.1 & 14.9 & 91.7 & 15.3 \\
3 & 90.3 & 15.8 & 89.3 & 16.5 \\
4 & 88.9 & 16.9 & 86.9 & 17.6 \\
5 & 87.5 & 17.7 & 84.9 & 18.6 \\
6 & 86.7 & 18.6 & 83.2 & 19.4 \\
7 & 85.8 & 19.7 & 81.8 & 20.8 \\
8 & 85.2 & 20.8 & 80.8 & 22.2 \\
9 & 84.7 & 21.9 & 79.9 & 23.3 \\
10 & 84.3 & 22.7 & 79.0 & 24.5 \\
11 & 84.0 & 23.6 & 78.3 & 25.5 \\
12 & 83.8 & 24.4 & 77.6 & 26.4 \\
\hline
\end{tabular}

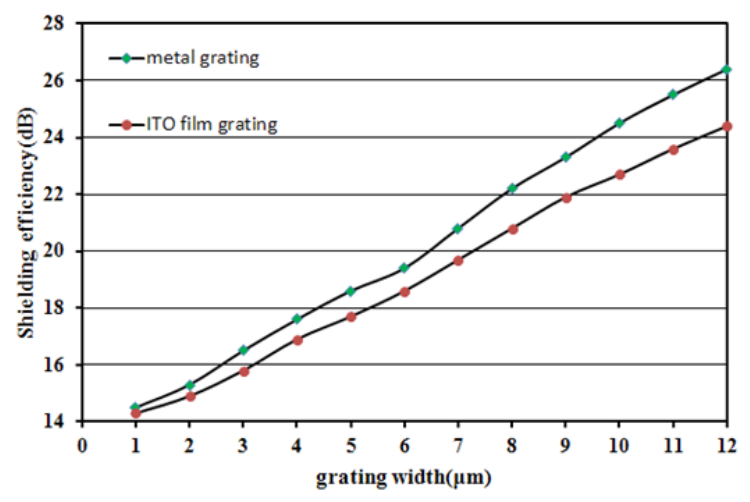

Figure 4. Shielding efficiency change law with grating width

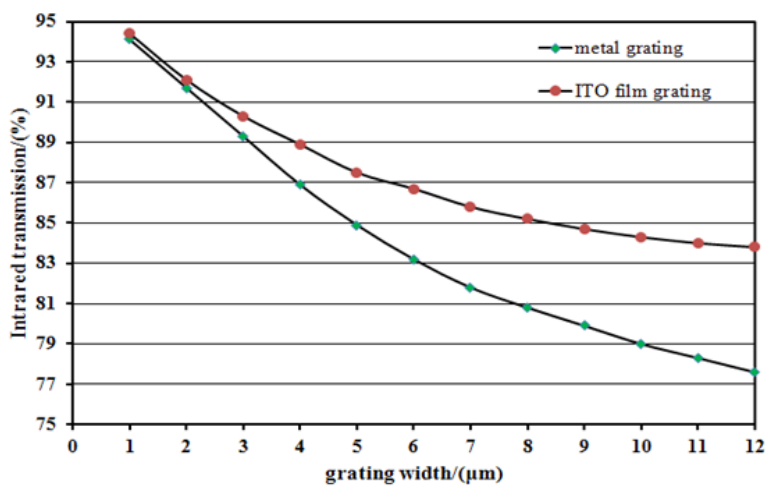

Figure 5. Intrared transmission change law with grating width

From Figure 4 and Figure 5 curve trend analysis, when mesh period g is constant, electromagnetic shielding effectiveness increases with the linewidth, infrared transmission decreases with increased width; curve from the two comparison mesh analysis available, metal mesh shielding effectiveness slightly ITO film mesh, but the 
difference is small, when the the line width is $10 \mu \mathrm{m}$, the shielding effectiveness of not more than $2 \mathrm{~dB}$, this small difference is due to the experiments of the obtained ITO film surface resistance slightly lower than the metal film, the problem can be improved by an appropriate increase in the film thickness. Infrared transmission aspect ITO film mesh above metal mesh, and quite different, when the width over $10 \mu \mathrm{m}$, a difference of $10 \%$ transmission.

\subsection{Transparent Conductive Mesh Cycle Affect Optical Properties}

To stuptical and electrical properties of the cycle, the experiment produced fixed width $6 \mu \mathrm{m}$, cycle $50 \sim 800 \mu \mathrm{m}$ two materials mesh, select the same $18 \mathrm{GHz}$ radar waves and infrared waves $4.6 \mu \mathrm{m}$ test results are listed in table 2. A quartz substrate infrared transmission of $95 \%$. Figures 6 and 7 in Table 2 obtained by visual graph.

Figures 6 and 7 from the curve trend analysis, when the line width $2 \mathrm{a}$ is constant, with the increase in period g, the mesh of the electromagnetic wave shielding efficiency decreases, and infrared transmission increased; likewise from the figure of two materials Comparative analysis mesh, metal mesh shielding efficiency greater than ITO film mesh, the difference is small, a difference of only $3 \mathrm{~dB}$ when cycle $50 \mu \mathrm{m}$. Infrared transmission aspect ITO mesh is greater than metal mesh, but the difference is greater, up $8 \%$ maximum.

Comprehensive analysis of experimental data: the line width increased to increase the shielding effectiveness, infrared transmission falling; Cycle increase shielding efficiency decline, and infrared transmission increased. Therefore, from the analysis of both periodic and width, no matter what selection parameters, high infrared transmission and high shielding effectiveness is a contradiction, and therefore should be considered in practical applications and environmental requirements, data from theoretical calculations and experimental reasonable choice the width of mesh and period.

\section{Results}

Paper Starting from the theoretical analyzes the relationship between the shielding effectiveness, infrared transmission and width and cycle, using magnetron sputtering prepared ITO transparent conductive films, and using the lithography and chemical erosion method make multiple pieces ITO film mesh. And using appropriate fabrication of metal mesh of the same structure. Through two grid test data statistics and analysis, it concluded: width increases with increased shielding efficiency of the mesh, infrared transmission decline; decline grid with periodic increases shielding effectiveness, infrared transmission increased. The same structure of the mesh, the higher the conductivity of shielding effectiveness better. Transparent conductive film mesh instead of metal mesh, infrared transmission has improved greatly, shielding efficiency small decline, so contradictory of visible / infrared optical window infrared transmission and shielding effectiveness between the transparent conductive film mesh to solve advantages. The transparent conductive film mesh in the visible / infrared optical window have more practical vaule.

Table 2. Different cycle shielding efficiency and infrared transmission

\begin{tabular}{ccccc}
\hline $\begin{array}{c}\text { Cycl/ } \\
\mu \mathrm{m}\end{array}$ & $\begin{array}{c}\text { ITO mesh Infrared } \\
\text { transmission } /(\%)\end{array}$ & $\begin{array}{c}\text { ITO mesh shielding } \\
\text { efficiency/dB }\end{array}$ & $\begin{array}{c}\text { Metal mesh Infrared } \\
\text { transmission } /(\%)\end{array}$ & $\begin{array}{c}\text { Metal mesh shielding } \\
\text { efficiency/dB }\end{array}$ \\
\hline & 73.2 & 38.0 & 66.1 & 41.0 \\
100 & 76.3 & 32.3 & 70.2 & 34.8 \\
150 & 79.4 & 28.3 & 73.8 & 30.4 \\
200 & 82.1 & 24.8 & 76.8 & 26.4 \\
250 & 84.4 & 21.7 & 79.9 & 23.0 \\
300 & 86.2 & 19.1 & 82.4 & 20.4 \\
350 & 87.8 & 17.2 & 84.8 & 18.0 \\
400 & 89.1 & 15.4 & 86.2 & 16.3 \\
450 & 90.3 & 14.3 & 87.8 & 15.1 \\
500 & 91.3 & 13.4 & 89.0 & 14.2 \\
550 & 92.3 & 12.6 & 90.1 & 13.4 \\
600 & 93.1 & 11.9 & 91.2 & 12.4 \\
650 & 93.8 & 11.4 & 92.2 & 11.9 \\
700 & 94.4 & 10.9 & 93.1 & 11.4 \\
750 & 94.7 & 10.5 & 93.9 & 10.7 \\
800 & 95 & 10.1 & 94.6 & 10.2 \\
\hline
\end{tabular}




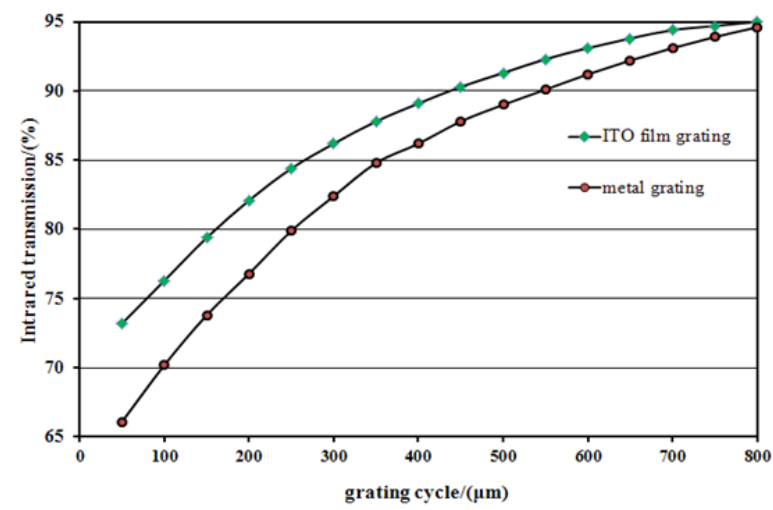

Figure 6. Intrared transmission change law with grating period

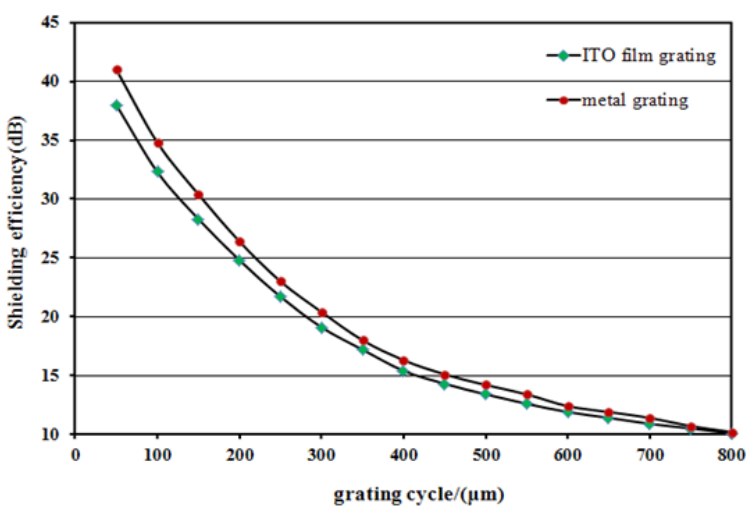

Figure 7. Shielding efficiency change law with grating period

\section{References}

Cranch, G. A., Flockhart, G. M. H., \& Kirken, C. K. (2008). IEEE Sens. J., 8, 1161.

Gazazyan, E. A., Papoyan, A. V., Sarkisyan, D., \& Weis, A. (2007). Laser Phys. Lett., 4, 801.

Guo, G., \& Sheng, Y. B. (2009). Research on laser direct writing system and its lithography properties. Proc. SPIE., 3550, 409-418.

Han, Y. L., Liu, D. S., \& Jiang, X. P. (2007). Sqare selffocu- sing lens array and its image. Acta Photonica Sinica, 36, 221.

Lin, F., \& Ruan, Y. Z. (2014). Analysis of FSS sandwiched in a dielectric sheet. Acta Aeronautica et Astronautica Sinic.

Munk, B. A. (2000). Frequency selective surfaces: theory and design. New York: JohnW iley.

Sonka, M., Hlavac, V., \& Boyle, R. (2003). Image Processing, Analysis, and Machine Vision. Posts \& Telecom Press, 11-15.

Wang, H. Q. (2001). Research on environment of electromagnetic radiation. Avionics Tech., 32(1), 29-34.

\section{Copyrights}

Copyright for this article is retained by the author(s), with first publication rights granted to the journal.

This is an open-access article distributed under the terms and conditions of the Creative Commons Attribution license (http://creativecommons.org/licenses/by/3.0/). 